\title{
Retraction Note to: Self-Lubricating Composites
}

\author{
Pradeep L. Menezes, Pradeep K. Rohatgi, and Emad Omrani
}

\section{Retraction Note to: \\ Chapter "Fundamentals of Solid Lubricants" in: P. L. Menezes et al. (eds.) Self-Lubricating Composites, https://doi.org/10.1007/978-3-662-56528-5_1}

The authors have retracted this chapter [1] because it significantly overlaps with a previously published article by Scharf and Prasad [2]. All authors agree with this retraction. [1] Prajapati A.K., Omrani E., Menezes P.L., Rohatgi P.K.: Fundamentals of Solid Lubricants. In: Menezes P., Rohatgi P., Omrani E. (eds.) Self-Lubricating Composites. Springer, Berlin, Heidelberg (2018) [2] Scharf, T.W., Prasad, S.V.: Solid lubricants: a review. J. Mater. Sci. 48, 511-531 (2013). https://doi.org/10. 1007/s10853-012-7038-2

\section{Retraction Note to: \\ Chapter "Self-Lubricating Polymer Composites" in: P. L. Menezes et al. (eds.) Self-Lubricating Composites, https://doi.org/10.1007/978-3-662-56528-5_3}

The authors have retracted this chapter [1] because it significantly overlaps with a previously published article by Fusaro [2]. All authors agree with this retraction. [1] Prajapati A.K., Omrani E., Menezes P.L., Rohatgi P.K.: Self-Lubricating Polymer Composites. In: Menezes P., Rohatgi P., Omrani E. (eds.) Self-Lubricating

The retracted version of this chapter can be found at https://doi.org/10.1007/978-3-662-56528-5_1 https://doi.org/10.1007/978-3-662-56528-5_3 
Composites. Springer, Berlin, Heidelberg (2018) [2] Fusaro, R.L.: Self-lubricating polymer composites and polymer transfer film lubrication for space applications, Tribology International, Volume 23, Issue 2, 105-122 (1990). https://doi.org/ $10.1016 / 0301-679 X(90) 90043-\mathrm{O}$ 